\title{
»Wo aber Gefahr ist, wächst das Rettende auch «? Die Internationalisierung von Risiken und die Entwicklung des völkerrechtlichen Katastrophenschutzrechts
}

\section{Einführung}

Welchen Beitrag leistet heute das Völkerrecht zur Verhütung und Bewältigung von Katastrophen? Diese Frage erscheint angesichts der Internationalisierung von Risiken, die zu Katastrophen führen können, unmittelbar plausibel. Sogleich denkt man an die Ölverschmutzung der Meere, an den Reaktorunfall in Tschernobyl und die Risiken der friedlichen Nutzung der Kernenergie, an den Klimawandel und wachsende Wirbelsturm- und Überschwemmungsgefahren. Doch beim weiteren Nachdenken über das Thema ergreift den Völkerrechtler auch ein Unbehagen: Ist es angemessen und fair, das Völkerrecht an seinen Erfolgen oder Mißerfolgen an dieser schwierigen und unsicheren Front zu messen? Ist die Katastrophe vielleicht für das Völkerrecht, was der Ausnahmezustand für das Staatsrecht ist? Noch befindet sich allerdings das Völkerrecht diesbezüglich nicht im Mittelpunkt der Kritik: Die öffentliche Meinung lastet ein Versagen bei der Verhütung und Bewältigung von Katastrophen, auch grenzüberschreitenden, nach wie vor primär den einzelnen Staaten und nicht der internationalen Gemeinschaft an - und dies, wie im folgenden ausgeführt werden wird, nicht ohne Grund.

Einen festen Begriff der »Katastrophe « hat das Völkerrecht bisher nicht ausgebildet. Die von der Bundesrepublik Deutschland mit ihren Nachbarstaaten und anderen Ländern abgeschlossenen Verträge über gegenseitige Hilfeleistung ${ }^{1}$ verwenden das Begriffspaar »Katastrophen und schwere Unglücksfälle« und nennen bestimmte Regelbeispiele. So heißt es in Art. 5 Abs. 1 des deutsch-österreichischen Abkommens von 1988, die Hilfe werde durch Hilfsmannschaften geleistet, »die insbesondere in der Bekämpfung von Bränden, von nuklearen und chemischen Gefahren und in Sanitätshilfe, Rettung, Bergung oder behelfsmäßiger Instandsetzung ausgebildet sind «. ${ }^{2}$ Im Rahmen der »Internationalen Strategie für Katastrophenreduzierung « der Vereinten Nationen ${ }^{3}$ wird der Begriff »disaster « definiert als »a serious disruption of the functioning of a community or a society causing widespread human, material, economic or environmental losses which exceed the ability of the affected community or society to cope using its own resources ${ }^{4} \ll$.

1 Vgl. zu diesen Verträgen unten Teile III.1.d und IV.1.a.

2 Abkommen zwischen der Bundesrepublik Deutschland und der Republik Österreich über die gegenseitige Hilfeleistung bei Katastrophen oder schweren Unglücksfällen vom 23.12.1988, BGB1. 1992 II S. 207. Das zeitlich letzte der zweiseitigen Abkommen, das deutsch-tschechische von 2000, verzichtet nunmehr allerdings auf solche Beispiele.

3 Vgl. unten Teil III 3. Vgl. auch Prinzip 2 der Erklärung von Rio.

4 Vgl. Inter-Agency Secretariat of the International Strategy for Disaster Reduction (UN/ISDR) (Hrsg.), Living with Risk: A global review of disaster reduction initiatives, 2004 version, Genf 2004, S. 17; im Internet abrufbar unter < http://www.unisdr.org/>. Dort auch eine Definition anderer zentraler Begriffe wie hazard, vulnerability, risk, prevention und preparedness. 
Der vorliegende Beitrag beschränkt sich auf einen Überblick über die Befassung des gegenwärtigen Völkerrechts mit Katastrophen: Welche Art von Katastrophen hat das Völkerrecht thematisiert, welche Schwerpunkte lassen sich erkennen? Welche Instrumente setzt es zu ihrer Verhütung und Bewältigung ein, welche Rolle spielen die internationalen Organisationen? Es soll versucht werden, das Material nach diesen Leitfragen zu ordnen und aus diesem Befund einige wertende Schlußfolgerungen zu ziehen. Zur Erleichterung der Übersicht wird zwischen Normen und Instrumenten danach unterschieden, ob sie der Verhinderung von Katastrophen dienen, also den Eintritt katastrophaler Ereignisse zu verhüten suchen (Teil III), oder ob sie die unmittelbare Bewältigung von Katastrophen im Falle ihres Eintritts (die Gefahrenabwehr) und den anschließenden Wiederaufbau zur Aufgabe haben (Teil IV). ${ }^{5}$ Eine Einzelanalyse der verschiedenen, zum Teil komplexen und sich wechselseitig ergänzenden Vertragswerke kann nicht geboten werden, ebensowenig eine empirisch begründete Beurteilung ihrer Effektivität. Ausgespart bleibt auch das Europarecht, obwohl sich für die Mitgliedstaaten der Europäischen Union heute eine komplexe Gemengelage völkerund europarechtlicher Verpflichtungen auch im Bereich der Katastrophenvorbeugung ergibt. $^{6}$

\section{Geschichtliche Voraussetzungen}

In den beiden großen deutschsprachigen Nachschlagewerken des Völkerrechts des 20. Jahrhunderts, dem »Wörterbuch des Völkerrechts und der Diplomatie « ${ }^{7}$ (1924-29) und dem »Wörterbuch des Völkerrechts $\aleph^{8}$ (1960-62), findet sich kein Eintrag über »Katastrophen « oder »Naturkatastrophen « im allgemeinen oder über »Dürre«, »Epidemie«, $»$ Erdbeben«, »Hungersnot « oder »Überschwemmung « im besonderen ${ }^{9}$. Der Umkehrschluß liegt nahe, daß solche Katastrophen und großen Unglücksfälle kein Thema

5 Allerdings werden in neueren Vertragswerken diese Sachbereiche auch gemeinsam behandelt; ein Beispiel ist das Internationale Übereinkommen vom 30.11.1990 über Vorsorge, Bekämpfung und Zusammenarbeit auf dem Gebiet der Ölverschmutzung (International Convention on Oil Pollution Preparedness, Response and Cooperation), BGB1. 1994 II S. 3799.

6 Vgl. für den Bereich des Schutzes gegen Öltankerunfälle: Karola Wolprecht, Die Rolle der Europäischen Union als besonderer Handlungsträger, in: Christian Tomuschat (Hrsg.), Schutz der Weltmeere gegen Öltankerunfälle - Das rechtliche Instrumentarium, Berlin 2005, S. 31ff. Vgl. auch die instruktive Mitteilung der Kommission »Stärkung der Reaktion der EU auf Katastrophen und Krisen in Drittländern« (KOM(2005) 153 endg., Ratsdok. 8382/05, Bundesratsdrucks. 312/05 vom 29.4.2005).

7 Begonnen von Julius Hatschek, fortgesetzt und hrsg. von Karl Strupp, 3 Bde., Berlin/Leipzig 1924-29.

8 Hrsg. von Hans-Jürgen Schlochauer (als »völlig neu bearbeitete zweite Auflage« des unter Fn. 7 genannten Werkes), 3 Bde. und Registerbd., Berlin 1960-62.

9 Allerdings erwähnt das Struppsche Wörterbuch in Bd. I, S. 574f., unter dem Stichwort »Internationale Verwaltungsgemeinschaften« die 1903 gegründete seismologische Union (Association internationale de sismologie): »Zweck der Union ist die Erdbebenforschung, deren Ergebnisse an der Hand der Berichte der einzelnen Stationen von einem in Straßburg errichteten Zentralbureau bearbeitet werden.« 
des klassischen Völkerrechts des 19. und der ersten Hälfte des 20. Jahrhunderts waren. Man wird hierfür zwei Hauptgründe namhaft machen können. Zum einen kamen Katastrophen dieser Art in Europa, auf das sich die Aufmerksamkeit der Völkerrechtslehre geographisch noch konzentrierte, nicht (mehr?) häufig vor. Vor allem aber wurden Katastrophenschutz und -bewältigung als Sache der einzelnen Staaten, nicht der Staatengemeinschaft betrachtet, und entsprechend in der Rechtsordnung als Aufgabe des nationalen, nicht des Völkerrechts. Dies wiederum hatte einen rechtlichen Grund, auf den sogleich eingegangen werden wird, nämlich die Verankerung der Völkerrechtsordnung im Souveränitätsprinzip, aber auch einen faktischen: Es waren die naturwissenschaftlich-technischen Voraussetzungen noch nicht gegeben, die es als möglich erscheinen ließen, den genannten Katastrophen durch internationale Zusammenarbeit und insbesondere völkerrechtliche Normierung wirksam zu begegnen.

Es intensivierte sich aber seit dem Beginn des 20. Jahrhunderts die völkerrechtliche Regelung von Gefahren in »staatsfreien Räumen «, insbesondere der Hohen See, wo die Staaten in Anbetracht des wachsenden Verkehrs etwa »die Nützlichkeit der Vereinbarung gewisser gemeinsamer Regeln über den Zusammenstoß von Schiffen« erkannten $^{10}$. Das Brüsseler Übereinkommen von 1910 regelte vor allem Haftungsfragen, kodifizierte aber auch eine allgemeine gegenseitige Beistandspflicht: »Nach einem Zusammenstoße von Schiffen ist der Kapitän jedes der Schiffe verpflichtet, dem anderen Schiffe und dessen Besatzung und Reisenden Beistand zu leisten (...)« (Art. 8 Abs. 1). Art. 11 des gleichzeitig abgeschlossenen Übereinkommens zur einheitlichen Feststellung von Regeln über die Hilfsleistung und Bergung in Seenot ${ }^{11}$ bestimmte: »Jeder Kapitän ist verpflichtet, allen Personen, selbst feindlichen, die auf See in Lebensgefahr angetroffen werden, Beistand zu leisten (...)«. Auch infolge solcher Regelungen sind die »Nichtstaatsgebiete« zu »Staatengemeinschaftsräumen« geworden, d.h. der Staatengemeinschaft insgesamt zugeordneten Räumen. ${ }^{12}$

\section{Staatliche Souveränität und das Prinzip nationaler Verantwortung für Katastro- phenschutz und-bewältigung}

Das Souveränitätsprinzip war der tragende Grundsatz des Völkerrechts des 19. und 20. Jahrhunderts. ${ }^{13} \gg$ Die Souveränität«, schrieb Otto Hintze, »bedeutet also: Herauslösung des Staates als eines Individuums aus der Gebundenheit alter Gemeinschaftsverhältnisse, Übergang zu individueller Selbstbestimmung. Voraussetzung dafür ist: Unabhängigkeit

10 So die Präambel des Brüsseler Übereinkommens zur einheitlichen Feststellung von Regeln über den Zusammenstoß von Schiffen vom 23.9.1910, RGB1. 1913 S. 49.

11 RGB1. 1913 S. 66. Das Übereinkommen regelte im übrigen den Anspruch auf Vergütung im Falle einer erfolgreichen Hilfsleistung oder Bergung. Art. 16 des Übereinkommens sah bereits eine periodische Überprüfung seiner Bestimmungen durch Konferenzen der Vertragsstaaten vor, »um etwaige Verbesserungen des Übereinkommens herbeizuführen und insbesondere sein Anwendungsgebiet, wenn möglich, zu erweitern«.

12 Vgl. Wolfgang Graf Vitzthum, Raum und Umwelt im Völkerrecht, in: ders. (Hrsg.), Völkerrecht, 3. Aufl. Berlin 2004, S. 357, 369ff., 399ff.

13 Vgl. Bardo Fassbender, Die Souveränität des Staates als Autonomie im Rahmen der völkerrechtlichen Verfassung. In: FS Erik Jayme, München 2004, S. $1089 \mathrm{ff}$. 
nach außen und Ausschließlichkeit der Staatsgewalt im Innern. $«{ }^{14}$ Der souveräne Staat der Neuzeit sei vor allem Machtstaat; »er muß sich in der neuen Staatengesellschaft behaupten können«. »[Es] herrscht durchaus der Grundsatz der Selbsthilfe der einzelnen Staaten. Jeder Staat muß sehen, wie er seine Interessen schützen und durchsetzen kann. ${ }^{15}$

Zwar bezog sich Hintze hier auf die außenpolitische und militärische Konkurrenz der europäischen Mächte, doch galt der »Grundsatz der Selbsthilfe« auch für die Bewältigung der vielgestaltigen Krisen, die sich auf dem Territorium eines Staates ereigneten. Denn, so sieht es die Staatslehre bis heute, der moderne Staat hat zu allererst die Sicherheit seiner Bürger zu gewährleisten: »Der erste, konstitutive Zweck des modernen Staates, von dessen Verwirklichung das Potential seiner sonstigen Zwecke abhängt, ist die Befriedung der Gesellschaft und die Herstellung des Gesamtzustandes der Sicherheit ${ }^{16}$. In der Legitimationsgeschichte des modernen Staates beziehe sich, schreibt Isensee, die Schutzpflicht zunächst auf das Leben (Hobbes), sodann auch auf Freiheit und Eigentum (Locke). »Außerhalb des vorstaatlich-naturrechtlich vorgegebenen Kernbereichs wird der Gegenstand der staatlichen Schutzpflichten bestimmt durch den jeweiligen Stand der rechtlichen und der zivilisatorischen Entwicklung. Die aktuelle ökologische Gefahrenlage, auf die der Staat der Gegenwart als Sicherheitsgarant antworten muß, ist eine Folge des technischen Fortschritts. « ${ }^{17}$

Doch blieben im modernen Völkerrecht bis in das frühe 19. Jahrhundert hinein naturrechtliche Anschauungen wirksam, die einerseits die Souveränität noch als eine beschränkte rechtliche Freiheit - und nicht, wie später, als eine Herrschaft über das Recht - verstanden, ${ }^{18}$ und andererseits zur Anerkennung einer internationalen Beistandspflicht im Katastrophenfalle führten. So schrieb Vattel in seinem berühmten und einflußreichen Völkerrechtslehrbuch von 1758 im Kapitel über die Pflichten einer Nation gegenüber den anderen Nationen, die auf ihrer gemeinsamen Zugehörigkeit zum Menschengeschlecht beruhen (Des Devoirs communs d'une Nation envers les autres, ou des Offices de l'humanité entre les Nations), es müsse eine Nation einem durch Hungersnot oder sonstiges schweres Unglück betroffenen Volk beistehen (elle doit assister un peuple désolé par la famine \& par d'autres calamités ${ }^{19}$ ): »Wenn ein Volk in-

14 Vgl. Otto Hintze, Wesen und Wandlung des modernen Staats (1931), in: ders., Staat und Verfassung. Gesammelte Abhandlungen zur allgemeinen Verfassungsgeschichte (hrsg. von Gerhard Oestreich), 2. Aufl. Göttingen 1962, S. 470 ff. (478).

15 Ebd. S. 479.

16 Vgl. Josef Isensee, Staat und Verfassung, in: Handbuch des Staatsrechts der Bundesrepublik Deutschland (HStR), Bd. II, 2004, § 15 Rn. 85. Vgl. auch dens., Gemeinwohl und Staatsaufgaben im Verfassungsstaat, in: HStR III, 1996 (1988), § 57 Rn. 44ff.: »Staatsziel Sicherheit und die Teleologie des Verfassungsstaates«.

17 Vgl. Isensee, Staat und Verfassung (Fn. 16), Rn. 91.

18 Vgl. Ulrich Scheuner, Die großen Friedensschlüsse als Grundlage der europäischen Staatenordnung zwischen 1648 und 1815, in: ders., Schriften zum Völkerrecht (hrsg. von Christian Tomuschat), Berlin 1984, S. 349ff. (373f.).

19 Vgl. Emer de Vattel, Le droit des gens ou principes de la loi naturelle, appliqués à la conduite et aux affaires des Nations et des Souverains, tom. I, London 1758, livre II, chap. I, $\S 5$, S. 260. 
folge Hungersnot in verzweifelter Lage ist, haben (...) alle Völker, die überschüssige Lebensmittelbestände haben, ihm in seiner Not beizustehen, ohne sich indessen selbst dem Mangel auszusetzen. (...) In der alleräußersten Not entspricht der Beistand so sehr der Menschlichkeit, daß man kaum eine zivilisierte Nation finden wird, die sich der Beistandspflicht völlig entzieht. (...) Auch in anderen Katastrophen [wie Brandkatastrophen], die über ein Volk hereinbrechen, schuldet man ihm den gleichen Beistand. $\ll^{20}$ Als zeitgenössisches Beispiel nannte Vattel die englische Hilfe nach dem Erdbeben von Lissabon (1755): »Bei der ersten Nachricht von dem Unglück von Lissabon bewilligte das Parlament eine Summe von hunderttausend Pfund Sterling für die Unterstützung des unglücklichen Volkes. Der König gab noch erhebliche Beträge dazu. Schiffe wurden schnellstens mit Vorräten und Hilfsmaterial jeder Art beladen. So wurden die Portugiesen davon überzeugt, daß die Verschiedenheit des Glaubensbekenntnisses und des Kultes niemals den abhält, der die Gebote der Menschlichkeit zu achten weiß. $\ll^{21}$ Allerdings sei, so Vattel, nur die um Hilfe angegangene Nation berechtigt darüber zu urteilen, ob ein wirkliches Bedürfnis vorliegt und ob es die Umstände, insbesondere die Rücksicht auf das eigene Wohl, ihr erlauben, die erwarteten Dienste zu bewilligen. Daher habe eine Nation nur ein unvollkommenes Recht auf die Dienste der Menschlichkeit; sie könne keine andere zwingen, sie ihr zu gewähren ${ }^{22}$.

Von einer solchen allgemeinen, wenngleich unvollkommenen Pflicht zur zwischenstaatlichen Hilfeleistung im Falle einer calamité war im 19. Jahrhundert keine Rede mehr. Erst nach dem Ersten und vor allem dem Zweiten Weltkrieg erfuhr sie eine Wiederbelebung im Zeichen der Idee der »internationalen Gemeinschaft«, die sich schrittweise institutionalisierte. ${ }^{23}$ Freilich war es den Staaten unbenommen, sich in besonderen Verträgen gegenseitigen Beistand zuzusagen.

\section{Die Pflicht zur Achtung der Unverletzlichkeit fremden Staatsgebietes}

Wenn nach dem Souveränitätsprinzip jeder Staat (ausschließlich) für die Verhütung und Bewältigung von Katastrophen auf seinem Staatsgebiet verantwortlich war, so ergab sich aus demselben Prinzip auch die Pflicht, die Unverletzlichkeit fremden Staatsgebiets zu achten, also nicht durch eigenes Handeln eine Katastrophe auf fremdem Gebiet zu verursachen oder das Übergreifen einer Katastrophe, die sich auf eigenem Gebiet ereignet hatte, auf fremdes Gebiet zu verantworten.

Es gelten diesbezüglich bis heute Grundsätze, wie sie im Jahre 1941 von einem amerikanisch-kanadischen Schiedsgericht im Trail Smelter-Fall formuliert wurden. Eine bei Trail am Columbia River in British Columbia gelegene Zink- und Bleischmelze ${ }^{24}$ hatte seit ihrer Vergrößerung im Jahre 1925 im US-Bundesstaat Washing-

20 Zit. nach der Übersetzung von Wilhelm Euler (Ausg. »Die Klassiker des Völkerrechts in modernen deutschen Übersetzungen«, hrsg. von Walter Schätzel, Bd. III, Tübingen 1959), S. 186.

21 Ebd.

22 Vgl. Vattel (Fn. 19), §§ 9 und 10, S. 264; in der Übersetzung von Euler (Fn. 20) S. 188.

23 Vgl. Ulrich Scheuner, Solidarität unter den Nationen als Grundsatz in der gegenwärtigen internationalen Gemeinschaft (1975), in: ders., Schriften (Fn. 18), S. 379ff. 
ton Schäden durch Schwefeldioxydgase verursacht. ${ }^{25}$ Das Schiedsgericht bejahte die Verpflichtung eines Staates, Ersatz für Schaden zu leisten, der in fremdem Staatsgebiet durch von eigenem Gebiet ausgehende Rauchentwicklung verursacht worden ist. Ein Staat sei auch verpflichtet, Vorkehrungen zur Verhinderung solcher Schäden zu treffen. ${ }^{26}$ Im Korfu Kanal-Fall ${ }^{27}$ bekräftigte der Internationale Gerichtshof (IGH) diese Grundsätze, indem er die völkerrechtliche Verpflichtung jedes Staates betonte »not to allow knowingly its territory to be used for acts contrary to the rights of other States ${ }^{28}$. Hieraus folge eine Pflicht des Gebietsstaates, andere Staaten von ihm bekannten Gefahren auf seinem Staatsgebiet zu unterrichten und vor ihnen zu warnen. In seinem Rechtsgutachten von 1996 über die Rechtmäßigkeit einer Drohung mit oder des Gebrauchs von Atomwaffen erklärte der IGH: »The existence of the general obligation of States to ensure that activities within their jurisdiction and control respect the environment of other States or of areas beyond national control is now part of the corpus of international law relating to the environment $\ll^{29}$.

Früh wurde jedoch erkannt, daß diese Prinzipien nationaler Verantwortlichkeit in sogenannten staatsfreien Räumen, d. h. Gebieten, die keiner einzelstaatlichen territorialen Souveränität unterstehen, nicht ausreichten. Nach dem Untergang des englischen Dampfers Titanic im April 1912 trat auf Initiative des Deutschen Kaisers im November 1913 in London eine Staatenkonferenz zusammen, um über gemeinsame Maßregeln zur Verhütung der Wiederkehr ähnlicher Katastrophen zu beraten. ${ }^{30}$ Die Konferenz beschloß am 20. Januar 1914 einen »Vertrag zum Schutze des menschlichen Lebens auf Hoher See«, der jedoch wegen des Ausbruchs des Weltkrieges nicht mehr ratifiziert wurde. Der Vertrag sah unter anderem die Einrichtung von Schiffen der Vertragsmächte zur Beobachtung der Eisverhältnisse im Nordatlantik vor, ferner Vorschriften über die Schiffskonstruktion von Passagierdampfern und die obligatorische Einrichtung der »Funkentelegraphie« auf Handelsschiffen, über Rettungsboote

24 smelter (engl.), Schmelzerei, Schmelzhütte.

25 Vgl. Peter Schneider, Trail Smelter-Fall, in: Wörterbuch des Völkerrechts (Fn. 8), Bd. 3, S. 447.

26 Vgl. den Schiedsspruch von 1941 in: American Journal of International Law Bd. 35 (1941), S. 716: »under principles of international law (...) no state has the right to use or permit the use of territory in such a manner as to cause injury by fumes in or to the territory of another or the properties or persons therein, when the case is of serious consequence and the injury is established by clear and convincing evidence."

27 Vgl. Rudolf Bernhardt, Korfu Kanal-Fall, in: Wörterbuch des Völkerrechts (Fn. 8), Bd. 2, S. $313 \mathrm{ff}$.

28 The Corfu Channel Case (Merits), Urteil vom 9.4.1949, ICJ Reports 1949, S. 4 (22).

29 Rechtsgutachten vom 8.7.1996, Abs. 29. Vgl. auch Prinzip 2 der Erklärung von Rio de Janeiro über Umwelt und Entwicklung vom 14. Juni 1992: »States have, in accordance with the Charter of the United Nations and the principles of international law, the sovereign right to exploit their own resources pursuant to their own environmental and developmental policies, and the responsibility to ensure that activities within their jurisdiction and control do not cause damage to the environment of other States or of areas beyond the limits of national jurisdiction. « Text der Erklärung in: International Legal Materials Bd. 31 (1992), S. 876, sowie in Christian Tomuschat (Hrsg.), Völkerrecht, 3. Aufl. Baden-Baden 2005, Nr. 25.

30 Vgl. Franz von Liszt, Völkerrecht, 12. Aufl. bearb. von Max Fleischmann, Berlin 1925, S. 368f. 
und -flöße sowie Maßnahmen zur Verhinderung, Entdeckung und Löschung von Schiffsbränden. Man kann in diesem Vertrag einen der ersten Schritte des Übergangs von einem System von völkerrechtlichen Verbotsnormen (Nichtschädigungsgeboten) zum Schutz jeweils einzelstaatlicher Rechtsgüter zu einem System der Kooperation (Handlungsgeboten) sehen, wie es heute insbesondere das Umweltvölkerrecht kennzeichnet.

\section{Krieg als Katastrophe und die Antwort des Völkerrechts}

Das Völkerrecht der Gegenwart ist durch die Erfahrung der beiden Weltkriege des 20. Jahrhunderts wesentlich geprägt. Insofern kann man sagen, daß der Krieg die »Urkatastrophe « ist, die zur heutigen Völkerrechtsordnung geführt hat. Die Charta der Vereinten Nationen vom 26. Juni 1945 wird mit der Erklärung eröffnet, die Völker der Vereinten Nationen seien fest entschlossen, »künftige Geschlechter vor der Geißel des Krieges zu bewahren, die zweimal zu unseren Lebzeiten unsagbares Leid über die Menschheit gebracht hat «. Entsprechend dienen das in der Charta stipulierte allgemeine zwischenstaatliche Gewaltverbot (Art. 2 Ziff. 4) und das Charta-System der kollektiven Sicherheit mit dem institutionellen Herzstück des Sicherheitsrates (Kap. V-VII) in erster Linie der Wahrung und - wenn nötig - Wiederherstellung des Weltfriedens. Die institutionellen Vorkehrungen der Völkerrechtsgemeinschaft gegen den Krieg haben als Vorbild für die Abwehr anderer Gefahren gedient.

Wenn hier vom Krieg als »Katastrophe« die Rede ist, so wird nicht verkannt, daß Kriege im Gegensatz zu Naturkatastrophen bewußt von Menschen geplant und ins Werk gesetzt werden. Doch sind die Grenzen fließend. Auch Kriege sind, selbst von den handelnden Politikern, als von Naturgewalten ausgelöst empfunden worden. »Alle hielten sich für die Angegriffenen, Könige, Diplomaten, Völker«, schrieb Golo Mann über den Beginn des Ersten Weltkriegs. ${ }^{31}$

Die Verfasser der UN-Charta erkannten bereits klar, daß der von ihnen angestrebte allgemeine Friede nicht allein auf der Friedensbereitschaft, dem guten Willen und der Völkerrechtstreue der Regierungen beruht, sondern von bestimmten weiteren Voraussetzungen abhängig ist. Als solche nennt die Präambel der Charta den »Glauben an die Grundrechte des Menschen, an Würde und Wert der menschlichen Persönlichkeit, an die Gleichberechtigung von Mann und Frau sowie von allen Nationen« und den »sozialen Fortschritt und einen besseren Lebensstandard in größerer Freiheit « sowie schließlich die Existenz »internationale(r) Einrichtungen (...), um den wirtschaftlichen und sozialen Fortschritt aller Völker zu fördern«. In Art. 1 Ziff. 3 der Charta wird als Ziel (purpose) der Vereinten Nationen beschrieben, »eine internationale Zusammenarbeit herbeizuführen, um internationale Probleme wirtschaftlicher, sozialer, kultureller und humanitärer Art zu lösen und die Achtung vor den Menschenrechten und Grundfreiheiten für alle ohne Unterschied der Rasse, des Geschlechts, der Sprache oder der Religion zu fördern und zu festigen« (vgl. auch Art. 55). In der Präambel der von der

31 Vgl. Golo Mann, Deutsche Geschichte des 19. und 20. Jahrhunderts, Ausg. Frankfurt/M. 1966, 1983, S. 590. 
UN-Generalversammlung im Dezember 1948 angenommenen Allgemeinen Erklärung der Menschenrechte heißt es entsprechend, die Anerkennung der angeborenen Würde und der gleichen und unveräußerlichen Rechte aller Mitglieder der Gemeinschaft der Menschen sei »die Grundlage von Freiheit, Gerechtigkeit und Frieden in der Welt«, und die Nichtanerkennung und Verachtung der Menschenrechte habe zu Akten der Barbarei (barbarous acts) geführt, die das Gewissen der Menschheit mit Empörung erfüllten.

Die Charta hat damit außer dem zwischenstaatlichen Krieg bereits andere Katastrophen in den Blick genommen: die systematische Unterdrückung von Menschenrechten und Grundfreiheiten einzelner, aber auch ethnisch, rassisch, sprachlich oder religiös bestimmter Gruppen, sowie elementare Armut und Not von Menschen und Völkern in Folge wirtschaftlicher Unterentwicklung und Benachteiligung (the equal rights of nations large and small). ${ }^{32}$ Mit der Konvention über die Verhütung und Bestrafung des Völkermordes von 1948 haben sich die UN-Mitgliedstaaten vor dem Hintergrund des Holocaust zum Ziel gesetzt, dieses spezifische Verbrechen, »das dem Geist und den Zielen der Vereinten Nationen zuwiderläuft und von der zivilisierten Welt verurteilt wird « (Präambel der Konvention), zu verhüten und zu bestrafen.

\section{Völkerrechtliche Normen und Instrumente zur Verhütung von nichtkriegerischen Katastrophen}

Die völkerrechtlichen Regelungen, die im vorliegenden Beitrag nur beispielhaft angeführt werden können, spiegeln die Erkenntnis der Regierungen, daß sich bestimmte Katastrophen heute wirksam nicht mehr allein im innerstaatlichen Rahmen, sondern nur durch internationale, oft institutionalisierte Zusammenarbeit der Staaten verhüten oder bewältigen lassen.

Im folgenden soll der Krieg als »Katastrophe« ausgeklammert werden ${ }^{3}, 3$ damit zugleich die Abkommen zur Verhinderung der Produktion, des Erwerbs, der Weitergabe und des Einsatzes und versuchsweisen Einsatzes bestimmter, als besonders gefährlich angesehener Waffen (insbesondere Kernwaffen, biologische und chemische Waffen), ferner die Verträge des humanitären Völkerrechts sowie diejenigen zum Schutz von Kulturgut in bewaffneten Konflikten.

Auch Tatbestände großer Bedeutung, die als »Katastrophen « im weiteren Sinne bezeichnet werden können und zum Gegenstand völkerrechtlicher Regelung gemacht worden sind - wie die Sklaverei, der Frauen- und Kinderhandel, die Zwangsarbeit oder die Gefährdung der Natur, einzelner Naturräume (z.B. der Alpen oder des Wattenmeers) oder bestimmter Arten freilebender Tiere und Pflanzen - bleiben unberücksichtigt. (Genannt sei aber das Übereinkommen zur Bekämpfung der Wüstenbildung von 1994, ${ }^{34}$ weil mit diesem ein Komplex zusammenhängender Umweltgefahren zum

32 Vgl. Bardo Fassbender, Der Schutz der Menschenrechte als zentraler Inhalt des völkerrechtlichen Gemeinwohls, EuGRZ 2003, S. 1ff.

33 Vgl. hierzu Bardo Fassbender, Die Gegenwartskrise des völkerrechtlichen Gewaltverbotes vor dem Hintergrund der geschichtlichen Entwicklung, EuGRZ 2004, S. $241 \mathrm{ff}$. 
Gegenstand einer völkerrechtlichen Regelung gemacht worden ist.) Dabei dienen die zahlreichen Abkommen zum Schutz der natürlichen Umwelt, der biologischen Vielfalt und des ökologischen Gleichgewichts indirekt auch der Verhütung von Katastrophen - ja letztlich sogar der »ultimativen Katastrophe«, nämlich einer Veränderung der ökologischen Bedingungen, welche die Fortsetzung menschlichen Lebens in Teilen der Erde oder auf der Erde überhaupt ausschlösse. Besondere Aufmerksamkeit haben in diesem Zusammenarbeit die Vereinbarungen zum Klimaschutz gefunden. ${ }^{35}$

Schließlich werden auch die Abkommen über Zusammenarbeit bei der Bekämpfung der organisierten Kriminalität und bestimmter Straftaten von erheblicher Bedeutung (wie des Handels mit Suchtstoffen oder der Geiselnahme) hier nicht behandelt, auch wenn diese der Verhütung von »Katastrophen« wie der Entführung von Flugzeugen durch sogenannte Selbstmordattentäter dienen ${ }^{36}$.

Nur punktuell angesprochen werden kann in diesem Beitrag die Frage der völkerrechtlichen Bekämpfung des Terrorismus, die in den vergangenen Jahren der Gegenstand zahlreicher Abhandlungen gewesen ist. ${ }^{37}$ In der Vergangenheit handelte es sich bei terroristisch motivierten Straftaten meistens um Attentate, Entführungen und Geiselnahmen einzelner Personen; diese Taten begründen nach einer Reihe von multilateralen Verträgen eine Verpflichtung der Staaten zur Verfolgung, Bestrafung und/oder Auslieferung. Doch haben neuere Anschläge, wie die des 11. September 2001 in den USA, die sich unterschiedslos gegen große Teile der Bevölkerung richten, katastrophische Ausmaße erreicht und gehören insofern zum Thema dieses Beitrags. Während für die verschiedenen Antiterrorismus-Konventionen und die Probleme der Zuständigkeit des UN-Sicherheitsrates nach Kapitel VII der UN-Charta sowie der Qualifizierung staatlicher Antiterrorismus-Maßnahmen als Selbstverteidigung im Sinne von Art. 51 der UN-Charta hier nur auf die Literatur verwiesen werden $\mathrm{kann}^{38}$, läßt sich für die unmittelbare Bewältigung terroristischer Gewaltakte durch zwischenstaatliche Zusam-

34 Übereinkommen der Vereinten Nationen vom 17.6.1994 zur Bekämpfung der Wüstenbildung in den von Dürre und/oder Wüstenbildung schwer betroffenen Ländern, insbesondere in Afrika, BGBl. 1997 II S. 1468. Vgl. hierzu zuletzt die Resolution 59/235 der UN-Generalversammlung vom 22.12.2004.

35 Vgl. das Übereinkommen vom 22.3.1985 zum Schutz der Ozonschicht, BGB1. 1988 II S. 901 (mit dem Montrealer Protokoll vom 16.9.1987 über Stoffe, die zu einem Abbau der Ozonschicht führen, BGBl. 1989 II S. 160, Neufassung BGBl. 2003 II S. 345), und das Rahmenübereinkommen der Vereinten Nationen vom 9.5.1992 über Klimaänderungen, BGB1. 1993 II S. 1783 (mit dem Protokoll von Kyoto vom 11.12.1997, BGB1. 2002 II S. 966). Vgl. hierzu zuletzt die Resolution 59/234 »Protection of global climate for present and future generations of mankind « der UN-Generalversammlung vom 22.12.2004.

36 Vgl. z.B. das Übereinkommen vom 16.12.1970 zur Bekämpfung der widerrechtlichen Inbesitznahme von Luftfahrzeugen, BGBl. 1972 II S. 1505, und das Übereinkommen vom 23.9.1971 zur Bekämpfung widerrechtlicher Handlungen gegen die Sicherheit der Zivilluftfahrt, BGBl. 1977 II S. 1229.

37 Aus der Fülle der Literatur nenne ich nur: Christian Tomuschat, Der 11. September 2001 und seine rechtlichen Konsequenzen, EuGRZ 2001, S. 535ff.; Andrea Bianchi (Hrsg.), Enforcing International Law Norms Against Terrorism, Oxford 2004; Christian Walter (Hrsg.), Terrorism as a Challenge for National and International Law: Security versus Liberty?, Berlin 2004. 
menarbeit (unten Teil IV) kurz feststellen, daß sich hierfür bisher noch kein völkerrechtliches Sonderrecht ausgebildet hat. So erfassen insbesondere die zweiseitigen Verträge über gegenseitige Hilfeleistung bei Katastrophen oder schweren Unglücksfällen $^{39}$ auch terroristische Gewalt, wenn sie von einer entsprechenden Größenordnung ist.

\section{Zwei- und mehrseitige Verträge}

a) Die älteste Schicht völkerrechtlicher Normen zur Verhinderung von (Natur-) Katastrophen bilden vermutlich zweiseitige Verträge unmittelbar benachbarter Staaten, in denen bestimmte Formen der Zusammenarbeit, darunter meist der Austausch relevanter Informationen und die Herstellung dauernder Verbindung zwischen den zuständigen Behörden, vereinbart werden, um den Eintritt von Katastrophen im Grenzgebiet (z.B. Überschwemmungen oder Verunreinigungen des Trinkwassers, aber auch Großbrände im Falle grenzüberschreitender Siedlungsgebiete) zu verhüten. ${ }^{40}$ Zeitweise hat die Literatur solche Regelungen einem »zwischenstaatlichen Nachbarrecht « zugeordnet. ${ }^{41}$ Dem Umstand, daß Luftverunreinigungen, die von einem Staat ausgehen, heute auch grenzferne Gebiete eines Nachbarstaates oder das Gebiet von Drittstaaten betreffen können, trägt ein Abkommen von $1979^{42}$ Rechnung. Die Erklärung von Rio de Janeiro fordert die Staaten auf, andere Staaten frühzeitig über Tätigkeiten zu informieren, die nachteilige grenzüberschreitende Umweltwirkungen haben könnten, und sich mit ihnen zu beraten; ${ }^{43}$ sie ist insofern Ausdruck des geltenden Völkergewohnheitsrechts.

b) $\mathrm{Zu}$ den frühen Verträgen gehören auch solche zur Verhütung von Unfällen auf gemeinsamen oder gemeinsam genutzten Verkehrswegen, wie den großen Flüssen und Weltmeeren. ${ }^{44}$ Hier reichte zur Gefahrenabwehr die jeweilige einzelstaatliche Juris-

38 Vgl. für die Frage des Sicherheitsrates etwa Bardo Fassbender, The UN Security Council and International Terrorism, in: Bianchi (Fn. 37), S. 83ff.

39 Vgl. unten Teil IV.1.a.

40 Vgl. z.B. das Übereinkommen vom 1.10 .1987 zwischen den Regierungen Deutschlands, Frankreichs und Luxemburgs über das Hochwassermeldewesen im Moseleinzugsgebiet, BGBl. 1988 II S. 93, sowie die Vereinbarung vom 17.9.1996 zwischen den Regierungen Deutschlands, Polens und der Tschechischen Republik über den Austausch von Immissionsdaten der Luftbelastung im »Schwarzen Dreieck«, BGB1. 1997 II S. 154.

41 Vgl. Hans Thalmann, Grundprinzipien des modernen zwischenstaatlichen Nachbarrechts, Zürich 1951, und den Artikel dess. über »Nachbarrecht, zwischenstaatliches« im Wörterbuch des Völkerrechts (Fn. 8), Bd. 2, S. 558ff. In ähnlicher Terminologie spricht Art. 24 Abs. 1a GG (eingefügt durch das Gesetz zur Änderung des Grundgesetzes vom 21.12.1992, BGBl. I S. 2086) von »grenznachbarschaftlichen Einrichtungen«.

42 Übereinkommen vom 13.11.1979 über weiträumige grenzüberschreitende Luftverunreinigung, BGBl. 1982 II S. 373 (vgl. für die weiteren Änderungen und Zusatzprotokolle den Fundstellennachweis B vom 4.2.2005, S. 646).

43 Vgl. Prinzip 19 der Erklärung (Fn. 29): »States shall provide prior and timely notification and relevant information to potentially affected States on activities that may have a significant adverse transboundary environmental effect and shall consult with those States at an early stage and in good faith.«

44 Vgl. z.B. die Revidierte Rheinschiffahrtsakte vom 17.10.1868, Preuß. GS 1869 S. 798, 836. 
diktion nicht aus, weil sich diese nur auf die eigenen Staatsangehörigen und Schiffe erstreckte, das Verhalten fremder Staatsangehöriger und die Sicherheit fremder Schiffe aber nicht steuern konnte. Die oben genannten Bemühungen von 1913/14 zum Schutz des menschlichen Lebens auf der Hohen See ${ }^{45}$ nahmen Vertragswerke von $1960,{ }^{46} 1972^{47}$ und $1974^{48}$ auf. Sie wurden im Rahmen der Internationalen Seeschiffahrts-Organisation (International Maritime Organization, IMO) erarbeitet, die für die Schiffssicherheit und den Meeresumweltschutz von zentraler Bedeutung ist. ${ }^{49}$

c) $\mathrm{Zu}$ den klassischen Themen internationaler Verträge zählt die Verhütung und gegebenenfalls gemeinsame Bekämpfung von grenzüberschreitenden Krankheiten, von denen Menschen, Tiere oder Pflanzen befallen werden können. Noch heute in Kraft ist das Internationale Abkommen vom 25. Juli 1934 über den gegenseitigen Schutz gegen das Denguefieber ${ }^{50}$ (auch Dandyfieber oder Siebentagefieber), eine tropische und subtropische Viruskrankheit, die durch Stechmücken übertragen wird. Durch ein Abkommen von 1924 wurde in Paris das Internationale Tierseuchenamt errichtet. ${ }^{51}$ Für die Bekämpfung der Maul- und Klauenseuche besteht eine eigene europäische Kommission. $^{52}$

d) Die zweiseitigen Verträge über die gegenseitige Hilfeleistung bei Katastrophen ${ }^{53}$ enthalten regelmäßig Vereinbarungen über eine Zusammenarbeit zur Katastrophenvorbeugung. So bestimmt Art. 11 Abs. 1 des deutsch-französischen Abkommens von 1977 ${ }^{54}$ : Um die Vorhersage, Vorbeugung und Bekämpfung von Katastrophen oder schweren Unglücksfällen zu fördern und zu verstärken, vereinbaren die Vertragsparteien eine ständige und enge Zusammenarbeit, indem sie alle zweckdienlichen Infor-

45 Vgl. oben bei Fn. 30.

46 Internationaler Schiffssicherheitsvertrag vom 17.6.1960 mit den zwei Teilen: a) Internationales Übereinkommen zum Schutz des menschlichen Lebens auf See, und b) Regeln zur Verhütung von Zusammenstößen auf See (Seestraßenordnung), BGBl. 1965 II S. 465, 742; 1966 II S. 1472.

47 Übereinkommen über die Internationalen Regeln zur Verhütung von Zusammenstößen auf See vom 20.10.1972, BGBl. 1976 II S. 1017.

48 Internationales Übereinkommen zum Schutz des menschlichen Lebens auf See vom 1.11.1974, BGBl. 1979 II S. 141; Protokoll von 1978 zu dem Übereinkommen: BGBl. 1980 II S. 525; Neufassung: BGBl. 1998 II S. 2579. Vgl. für die weiteren Änderungen den Fundstellennachweis B vom 4.2.2005, S. 622. Vgl. auch das Internationale Übereinkommen vom 27.4.1979 über den Such- und Rettungsdienst auf See, BGBl. 1982 II S. 485.

49 Vgl. das Übereinkommen vom 6.3.1948, BGBl. 2002 II S. 1870, und die auf dieser Grundlage beschlossenen Verträge und Vorschriften. Vgl. Christian Tomuschat, Die Internationale Seeschiffahrts-Organisation als Hauptakteur, in: ders., Schutz der Weltmeere (Fn. 6), S. $21 \mathrm{ff}$.

50 RGBl. 1936 II S. 235.

51 Vgl. das Internationale Übereinkommen vom 25.1.1924 zur Errichtung eines Internationalen Tierseuchenamts in Paris, RGBl. 1928 II S. 317, BGBl. 1974 II S. 676.

52 Vgl. die Satzung der Europäischen Kommission zur Bekämpfung der Maul- und Klauenseuche vom 11.12.1953, BGBl. 1975 II S. 625.

53 Vgl. dazu unten Teil IV.1.a.

54 Abkommen zwischen der Bundesrepublik Deutschland und der Französischen Republik über die gegenseitige Hilfeleistung bei Katastrophen oder schweren Unglücksfällen vom 3.2.1977, BGBl. 1980 II S. 34. 
mationen wissenschaftlich-technischer Art austauschen und regelmäßige Tagungen (réunions périodiques) vorsehen «. In neueren Abkommen werden zusätzlich Studienaufenthalte für Fachkräfte, gemeinsame Forschungsprogramme und Fachkurse, die Zusammenarbeit von Ausbildungseinrichtungen sowie gemeinsame Übungen vorgesehen. ${ }^{55}$ Außerdem verpflichten sich die Vertragsparteien $\mathrm{zu}$ einem $\gg$ Austausch von Informationen über Gefahren und Folgen von Katastrophen und schweren Unglücksfällen, die sich auf das Hoheitsgebiet des anderen Vertragsstaates ausbreiten können « (insbesondere zu einem Austausch von Meßdaten), sowie zu einer Untersuchung der Ursachen von Katastrophen und schweren Unglücksfällen ${ }^{56}$.

e) Hervorzuheben sind die zahlreichen Verträge zum Schutz von grenzüberschreitenden Flüssen (wie des Rheins ${ }^{57}$, der Donau ${ }^{58}$ und der Maas ${ }^{59}$ ) und der Meere ${ }^{60}$ vor Verunreinigung, insbesondere einer solchen durch Öl. ${ }^{61}$ Im Falle der Mosel, der Saar, des Rheins, der Elbe, der Oder und der Maas sind eigene internationale Kommissionen errichtet worden. ${ }^{62}$ Hier zeigt ein Vergleich der die Mosel (1961) und die Maas (2002) betreffenden Verträge eindrucksvoll den Fortschritt der Rechtsentwicklung, auch im Rahmen der Europäischen Union. Beschränkte sich das Mosel-Protokoll in seinem

55 Vgl. z.B. Art. 13 Abs. 1 Buchst. b des Vertrags zwischen der Bundesrepublik Deutschland und der Tschechischen Republik über die gegenseitige Hilfeleistung bei Katastrophen und schweren Unglücksfällen vom 19.9.2000, BGBl. 2002 II S. 1875.

56 Vgl. z.B. Art. 13 Abs. 1 Buchst. c des deutsch-österreichischen Abkommens von 1988 (Fn. 2) und Art. 13 Abs. 1 Buchst. c und d des deutsch-tschechischen Vertrags von 2000 (Fn. 55).

57 Vgl. das Übereinkommen vom 3.12.1976 zum Schutz des Rheins gegen chemische Verunreinigung, BGBl. 1978 II S. 1053, das Übereinkommen vom 3.12.1976 zum Schutz des Rheins gegen Verunreinigung durch Chloride, BGBl. 1978 II S. 1053, 1065, und das Übereinkommen vom 12.4.1999 zum Schutz des Rheins, BGB1. 2001 II S. 849.

58 Vgl. Übereinkommen vom 29.6.1994 über die Zusammenarbeit zum Schutz und zur verträglichen Nutzung der Donau, BGBl. 1996 II S. 874.

59 Vgl. Internationales Maasübereinkommen vom 3.12.2002, BGBl. 2004 II S. 1181.

60 Vgl. das Internationale Übereinkommen zur Verhütung der Verschmutzung der See durch Öl von 1954 (BGBl. 1956 II S. 379), das Übereinkommen vom 29.12.1972 über die Verhütung der Meeresverschmutzung durch das Einbringen von Abfällen und anderen Stoffen (BGB1. 1977 II S. 165, 180), das Internationale Übereinkommen vom 2.11.1973 zur Verhütung der Meeresverschmutzung durch Schiffe und das Protokoll vom 17.2.1978 zu diesem Übereinkommen (»MARPOL 73/78«) (BGBl. 1984 II S. 231; 1996 II S. 399, vgl. für die weiteren Änderungen den Fundstellennachweis B vom 4.2.2005, S. 614), das Übereinkommen vom 4.6.1974 zur Verhütung der Meeresverschmutzung vom Lande aus (BGBl. 1981 II S. 870), und Art. 207ff. des Seerechtsübereinkommens der Vereinten Nationen vom 10.12.1982 (BGBl. 1994 II S. 1798).

61 Vgl. auch Graf Vitzthum (Fn. 12), S. 433 ff., und Wolff Heintschel von Heinegg, Internationales öffentliches Umweltrecht, in: Knut Ipsen, Völkerrecht, 5. Aufl. München 2004, S. 973, 985ff. (Verträge gegen die Verschmutzung internationaler Binnengewässer), 993ff. (Schutz der Meeresumwelt).

62 Internationale Kommission zum Schutz der Mosel gegen Verunreinigung (Protokoll vom 20.12.1961, BGBl. 1962 II S. 1102); Internationale Kommission zum Schutz der Saar gegen Verunreinigung (Protokoll vom 20.12.1961, BGB1. 1962 II S. 1106); Internationale Kommission zum Schutz des Rheins gegen Verunreinigung (Vereinbarung vom 29.4.1963, BGBl. 1965 II S. 1432); Internationale Kommission zum Schutz der Elbe (Vereinbarung vom 8.10.1990, BGBl. 1992 II S. 942); Internationale Kommission zum Schutz der Oder gegen Verunreinigung (Vertrag vom 11.4.1996, BGBl. 1997 II S. 1707). 
Art. 2 noch auf eine ganz allgemeine Bestimmung der Aufgaben der Kommission (»kann den unterzeichneten Regierungen alle geeigneten Maßnahmen zum Schutz der Mosel gegen Verunreinigung vorschlagen«), ist das Maasübereinkommen zu einer detaillierten Beschreibung der Ziele und Grundsätze der Zusammenarbeit sowie der Aufgaben der Kommission übergegangen. So bekennen sich die Vertragsparteien zu den leitenden Grundsätzen der Vorsorge und Vorbeugung, dem Ursprungs- und Verursacherprinzip (Art. 3). Die Vertragsparteien verpflichten sich unter anderem zusammenzuarbeiten, um »Maßnahmen zur Vorbeugung und zum Schutz gegen Überschwemmungen unter Berücksichtigung der ökologischen Aspekte, der Raumordnung, der Landschaftspflege und anderer Bereiche (...) zu koordinieren und um auch durch Vorbeugungsmaßnahmen - zur Minderung der Auswirkungen von Überschwemmungen und Dürren beizutragen« (Art. 2 Buchst. c).

f) Die mit der friedlichen Nutzung der Atomenergie verbundenen Gefahren sind der Gegenstand weiterer bilateraler ${ }^{63}$ und multilateraler Abkommen ${ }^{64}$. Die bilateralen Abkommen stipulieren in der Regel eine Verpflichtung, die andere Vertragspartei möglichst frühzeitig über nukleare Unfälle zu informieren sowie einen kontinuierlichen Informations- und Erfahrungsaustausch über atomare Anlagen, kerntechnische Sicherheit und Strahlenschutz zu unterhalten. Als gefährlich werden auch bestimmte nicht-radioaktive Abfälle eingestuft. ${ }^{65}$

\section{Internationale Organisationen}

Zwischen vielen der im Vorabschnitt genannten mehrseitigen Verträge und internationalen zwischenstaatlichen Organisationen besteht eine Verbindung in der Weise, daß die Verträge von den Organen solcher Organisationen oder unter ihrer Schirmherrschaft erarbeitet oder initiiert worden sind, oder Zuständigkeiten internationaler Organisationen für ihre Durchführung und Überwachung begründen.

Im übrigen werden internationalen Organisationen auch durch ihre Gründungsverträge (Satzungen) Aufgaben der Verhütung von Katastrophen zugewiesen. Es können hierfür nur einige Beispiele angeführt werden. Bereits erwähnt wurde die zentrale Rolle der Internationalen Seeschiffahrts-Organisation (IMO) für die Schiffssicherheit und den Meeresumweltschutz. ${ }^{66}$

63 Vgl. die umfassende Liste solcher Verträge (nach dem Stand vom 31.10.1991) in: Günther Handl, Grenzüberschreitendes nukleares Risiko und völkerrechtlicher Schutzanspruch, Berlin 1992, S. 123ff.

64 Vgl. die Satzung der Internationalen Atomenergie-Organisation vom 26.10.1956, BGBl. 1957 II S. 1357, das Übereinkommen vom 20.9.1994 über nukleare Sicherheit, BGBl. 1997 II S. 130, und das Gemeinsame Übereinkommen vom 5.9.1997 über die Sicherheit der Behandlung abgebrannter Brennelemente und über die Sicherheit der Behandlung radioaktiver Abfälle, BGBl. 1998 II S. 1752.

65 Vgl. das Basler Übereinkommen vom 22.3.1989 über die Kontrolle der grenzüberschreitenden Verbringung gefährlicher Abfälle und ihrer Entsorgung, BGBl. 1994 II S. 2703, und das Übereinkommen vom 17.3.1992 über die grenzüberschreitenden Auswirkungen von Industrieabfällen, BGBl. 1998 II S. 1527.

66 Vgl. oben Teil III.1.b. 
Zu den Aufgaben der Weltgesundheitsorganisation (WHO) ${ }^{67}$ gehört die Bekämpfung und Ausrottung epidemischer, endemischer und sonstiger Krankheiten. Um diesen Zweck zu erreichen, leitet und koordiniert die WHO die internationale Beratung von Gesundheitsfragen, unterstützt die Regierungen der Mitgliedstaaten auf deren Ersuchen beim Ausbau ihrer öffentlichen Gesundheits- und Fürsorgedienste, fördert die medizinische Ausbildung und wissenschaftliche Forschung auf den Gebieten der Medizin, Hygiene und des öffentlichen Gesundheitswesens, erarbeitet internationale Normen für Arzneimittel, organisiert globale Impfprogramme, stellt Gesundheitsstatistiken auf und unterhält einen Nachrichtendienst. Mit den im Mai 2005 von der WHO-Weltgesundheitsversammlung angenommenen revidierten Internationalen Gesundheitsvorschriften (International Health Regulations) ${ }^{68}$ die im Mai 2007 in Kraft treten werden, sind die entsprechenden Verpflichtungen der Mitgliedstaaten und die Handlungsmöglichkeiten der WHO erheblich verstärkt worden. Zu den derzeitigen Arbeitsschwerpunkten der WHO gehören die Bekämpfung und Behandlung von HIV/AIDS (die $» 3$ by 5-Initiative« strebt an, bis Ende 2005 drei Millionen HIV-Infizierte in Entwicklungsländern mit antiretroviralen Medikamenten zu behandeln) und die weltweite Ausrottung von Poliomyelitis. In ihrer europäischen Region konzentriert sich die WHO gegenwärtig auf die Bekämpfung der Tuberkulose, des Alkoholmißbrauchs und des Tabakkonsums.

Die Ernährungs- und Landwirtschaftsorganisation der Vereinten Nationen (FAO) hat die Aufgabe, »die Menschheit vom Hunger zu befreien $\aleph^{69}$, und zu diesem Zweck die Land-, Forst- und Fischereiwirtschaft der Mitgliedstaaten zu unterstützen und zu verbessern.

Als Beispiel einer Zusammenarbeit internationaler Organisationen bei der Verhütung von Katastrophen sei die Entwicklung einer »globalen Strategie« zur Kontrolle der Vogelgrippe durch die FAO, die WHO und die Weltorganisation für Tiergesundheit (Office International des Epizooties, OIE) in Paris genannt. ${ }^{70}$

$\mathrm{Zu}$ den Aufgaben der Weltorganisation für Meteorologi ${ }^{1}{ }^{1}$ gehören die Förderung der weltweiten Zusammenarbeit bei der Einrichtung von Stationsnetzen für meteorologische Beobachtungen sowie die Unterstützung bei der Errichtung und dem Betrieb von zentralen meteorologischen Dienstleistungseinrichtungen. Die Weltorganisation unterstützt die Arbeit der nationalen meteorologischen Einrichtungen, etwa bei der meteorologischen Sicherung der Luft- und Seefahrt, der Herausgabe von amtlichen Warnungen über Wettererscheinungen, der kurz- und langfristigen Erfassung, Über-

67 Vgl. Art. 2 der Satzung der Weltgesundheitsorganisation vom 22.7.1946, BGBl. 1974 II S. 45.

68 Revision of the International Health Regulations, $58^{\text {th }}$ World Health Assembly, WHA58.3, Agenda Item 13.1 (23.5.2005), im Internet abrufbar unter $<$ http://www.who.int/csr/ihr/en/>. Kurze Zusammenfassung der Änderungen bei: Gerald S. Schatz, International Health Regulations: New Mandate for Scientific Cooperation, ASIL Insight vom 2.8.2005 (hrsg. von der American Society of International Law).

69 Präambel der FAO-Satzung vom 16.10.1945, BGB1. 1971 II S. 1036.

70 Vgl. »A Global Strategy for the Progressive Control of Highly Pathogenic Avian Influenza (HPAI)«, Mai 2005.

71 Vgl. das Übereinkommen vom 11.10.1947, BGBl. 1990 II S. 171. Vgl. auch das Europäische Zentrum für mittelfristige Wettervorhersage (Übereinkommen vom 11.10.1973, BGBl. 1975 II S. 873). 
wachung und Bewertung der meteorologischen Prozesse sowie der Vorhersage der meteorologischen Vorgänge. ${ }^{72}$ Mit dieser Arbeit sollen auch bevorstehende extreme Wetterereignisse rechtzeitig erkannt und ihre negativen Folgen für die Bevölkerung gemildert werden. ${ }^{73}$

Der Sicherheit der zivilen Luftfahrt dient die Arbeit der Internationalen Zivilluftfahrt-Organisation (ICAO), die mit dem Abkommen über die Internationale Zivilluftfahrt vom 7. Dezember 1944 (ICAO-Abkommen oder Konvention von Chicago) ${ }^{74}$ gegründet wurde. Die Organisation hat die Aufgabe, einheitliche Regelungen für die Sicherheit, Regelmäßigkeit und Wirtschaftlichkeit des internationalen Luftverkehrs zu erarbeiten und im Einklang mit dem technischen Fortschritt und den Erfordernissen des Verkehrs weiterzuentwickeln, darunter etwa Regeln über Betrieb und Abwicklung des Luftverkehrs, Nachrichtenübermittlung, Eintragung und Kennzeichnung von Luftfahrzeugen, Lufttüchtigkeit des Luftfahrtgeräts und die Luftsicherheit (vgl. Art. 44 des ICAO-Abkommens). Die von der ICAO auf der Grundlage von Art. 37 des Abkommens beschlossenen Richtlinien (Standards) und Empfehlungen (Recommendations) sind zum überwiegenden Teil in den Anhängen zum Abkommen enthalten. Sie gelten nicht unmittelbar in den Mitgliedstaaten, sondern müssen von diesen in nationales Recht umgesetzt werden.

Nicht eingegangen werden kann hier auf die zahlreichen Organisationen, Institutionen und Programme innerhalb und außerhalb des UN-Systems, die sich der Entwicklungszusammenarbeit, der Armutsbekämpfung, dem Umwelt- und Menschenrechtsschutz widmen, obwohl ihre langfristige und kontinuierliche Arbeit selbstredend auch Katastrophen verhindern soll, ja für die Verhütung von Katastrophen unverzichtbar ist.

\section{Naturkatastrophen als Schwerpunkt der Arbeit der Vereinten Nationen}

Die Vereinten Nationen bemühen sich in verschiedenen Organen und Organisationen des UN-Systems um eine Verminderung der Zahl von Naturkatastrophen und eine Begrenzung ihrer negativen Folgen. ${ }^{75}$ Die UN-Generalversammlung erklärte das Jahrzehnt von 1990 bis 1999 zur »International Decade for Natural Disaster Reduction«. Im Mai 1994 fand in Yokohama, Japan, die UN-Weltkonferenz zur Reduzierung von Naturkatastrophen statt; sie verabschiedete die »Yokohama Principles, Strategy and

72 Vgl. die Aufgaben des Deutschen Wetterdienstes gem. § 4 Gesetz über den Deutschen Wetterdienst (DWD-Gesetz), BGBl. 1998 I S. 2871.

73 Vgl. auch § 4 Abs. 4 DWD-Gesetz: »Im Rahmen seiner Aufgaben nach Absatz 1 unterstützt der Deutsche Wetterdienst die Länder bei der Durchführung ihrer Aufgaben im Bereich des Katastrophenschutzes, insbesondere bei extremen Wetterereignissen, und beteiligt sich an den Aufgaben im Rahmen der Zivilen Verteidigung und der zivil-militärischen Zusammenarbeit.«

74 BGBl. 1956 II S. 411.

75 Vgl. für das folgende: Living with Risk (Fn. 4). Vgl. auch United Nations Development Programme (UNDP) - Bureau for Crisis Prevention and Recovery (Hrsg.), Reducing Disaster Risk: A Challenge for Development, New York 2004, sowie Bundesministerium für wirtschaftliche Zusammenarbeit und Entwicklung (Hrsg.), Katastrophenvorsorge - Beiträge der deutschen Entwicklungszusammenarbeit (Reihe »Materialien«, Nr. 135), Bonn 2004. 
Plan of Action for a Safer World«. Die Prinzipienerklärung bekräftigte die primäre Verantwortung jedes einzelnen Staates für den Schutz seiner Bevölkerung und Infrastruktur vor Naturkatastrophen.

Im Jahr 2000 beschloß die UN-Generalversammlung die »Internationale Strategie für Katastrophenreduzierung « (International Strategy for Disaster Reduction, ISDR ${ }^{76}$ und richtete in Genf ein Sekretariat (Inter-Agency Secretariat) und eine Inter-Agency Task Force für Katastrophenreduzierung ein. Unter dem Vorsitz des UN-Untergeneralsekretärs für humanitäre Angelegenheiten gehören der Task Force Vertreter verschiedener UN-Organisationen und Einrichtungen, regionaler Organisationen und der Zivilgesellschaft an. Im Herbst 2003 war die Bundesregierung Gastgeberin einer internationalen Frühwarnkonferenz in Bonn.

Im Januar 2005 fand in Kobe in der japanischen Provinz Hyogo unter Beteiligung von 154 Staaten die UN-Weltkonferenz zur Reduzierung von Katastrophen (World Conference on Disaster Reduction) statt. Sie beschloß die »Erklärung von Hyogo « und den »Hyogo-Aktionsrahmen für 2005-2015« (Hyogo Framework for Action 20052015: Building the Resilience of Nations and Communities to Disasters). ${ }^{77}$ Die Erklärung hebt besonders den Zusammenhang zwischen wirtschaftlicher und sozialer Entwicklung und Katastrophenschutz hervor: Naturkatastrophen machten in kürzester Zeit Entwicklungsanstrengungen und -investitionen zunichte, und stellten ein großes Hindernis auf dem Weg zu nachhaltiger Entwicklung und Armutsbeseitigung dar. »Coping with and reducing disasters so as to enable and strengthen nations' sustainable development is, therefore, one of the most critical challenges facing the international community « (Präambel). Die Erklärung stellt schlicht fest, daß menschliche Gesellschaften mit den von der Natur ausgehenden Risiken leben müßten. Sie seien aber nicht zur Untätigkeit verurteilt, sondern könnten sich auf Katastrophen vorbereiten und ihre Folgen lindern. Insbesondere könne und müsse die Verletzlichkeit (vulnerability) von Gesellschaften reduziert werden (Ziff. 3 der Erklärung).

Die hier kurz dargestellte Arbeit der Vereinten Nationen steht in einem engen Zusammenhang mit ihren langjährigen Bemühungen um die Anerkennung eines Menschenrechts auf Entwicklung (right to development) ${ }^{78}$ und eines Rechts auf Nahrung (right to food) ${ }^{79}$.

76 Vgl. hierzu zuletzt die Resolutionen 59/212 und 59/231 der UN-Generalversammlung vom 20. und 22.12.2004 und den Bericht des UN-Generalsekretärs vom 1.8.2005, UN-Dok. A/ $60 / 180$.

77 Vgl. Report of the World Conference on Disaster Reduction, UN-Dok. A/CONF.206/6 vom 16.3.2005.

78 Vgl. hierzu zuletzt die Resolution 59/185 der UN-Generalversammlung vom 20.12.2004.

79 Vgl. hierzu zuletzt die Resolution 59/202 der UN-Generalversammlung vom 20.12.2004. Vgl. auch die Resolution 59/186 über »Menschenrechte und extreme Armut« vom 20.12.2004. 


\section{Völkerrechtliche Normen und Instrumente zur unmittelbaren Bewältigung von nichtkriegerischen Katastrophen und zur Wiederaufbauhilfe}

\section{Zwei- und mehrseitige Verträge}

a) In zahlreichen zweiseitigen Verträgen haben sich - in der Regel benachbarte - Staaten gegenseitige Hilfeleistung bei Katastrophen oder schweren Unglücksfällen zugesagt. Die Bundesrepublik Deutschland hat, teilweise unter Ersetzung älterer Verträge, seit Mitte der 1970er Jahre entsprechende Abkommen mit ihren Nachbarstaaten Belgien, Dänemark, Frankreich, Luxemburg, den Niederlanden, Österreich, Polen, der Schweiz, der Tschechischen Republik und Ungarn geschlossen, ${ }^{80}$ außerdem mit Litauen und der Russischen Föderation ${ }^{81}$. Im Verhältnis zu Frankreich besteht eine weitere Vereinbarung über Informationsaustausch bei Vorkommnissen oder Unfällen, die radiologische Auswirkungen haben können, von $1981^{82}$, im Verhältnis zur Schweiz ein Abkommen über die Erleichterung von Rettungseinsätzen und Rücktransporten mit Luftfahrzeugen von $1965^{83}$ sowie eine Vereinbarung über den radiologischen Notfallschutz von $1978^{84}$.

Die Verträge enthalten typischerweise Bestimmungen über die Behörden, welche für die Anforderung und Auslösung der Hilfsmaßnahmen zuständig sind, über die Erleichterung des Grenzübertritts der Hilfsmannschaften und des Grenzübergangs des Materials, über Einsätze mit Luftfahrzeugen sowie über Einsatzkosten, Schadensersatz und Entschädigung. Ferner wird eine Zusammenarbeit zur Vorbeugung und Bekämpfung von Katastrophen vereinbart. ${ }^{85}$ In einzelnen Verträgen ${ }^{86}$ wird Personen, die als Helfer oder Evakuierte von einem Vertragsstaat in den anderen gelangt sind, Sozialhilfe bis zum Zeitpunkt der frühesten Rückkehrmöglichkeit zugesagt. Neuerdings werden in die Verträge auch Bestimmungen zum Schutz personenbezogener Daten aufgenommen. ${ }^{87}$

b) In der Tradition der älteren völkerrechtlichen Regeln über die Pflicht zum Beistand bei Schiffszusammenstößen und im Falle der Seenot ${ }^{88}$ verpflichtet etwa das Abkom-

80 Vgl. die Abkommen über gegenseitige Hilfeleistung bei Katastrophen oder schweren Unglücksfällen mit Belgien vom 6.11.1980 (BGBl. 1982 II S. 1006), Dänemark vom 16.5.1985 (1988 II S. 286), Frankreich vom 3.2.1977 (1980 II S. 33), Luxemburg vom 2.3.1978 (1981 II S. 445), den Niederlanden vom 7.6.1988 (1992 II S. 198), Österreich vom 23.12.1988 (1992 II S. 206), Polen vom 10.4.1997 (1998 II S. 1178), der Schweiz vom 28.11.1984 (1987 II S. 74), der Tschechischen Republik vom 19.9.2000 (2002 II S. 1874) und Ungarn vom 9.6.1997 (1998 II S. 1189).

$81 \mathrm{Vgl}$. die Abkommen über gegenseitige Hilfeleistung bei Katastrophen oder schweren Unglücksfällen mit Litauen vom 15.3.1994 (BGBl. 1996 II S. 27) und der Russischen Föderation vom 16.12.1992 (1994 II S. 3542).

82 Vereinbarung vom 28.1.1981, BGBl. 1981 II S. 885.

83 Abkommen vom 29.4.1965, BGBl. 1967 II S. 773.

84 Vereinbarung vom 31.5.1978, BGBl. 1980 II S. 563; 1988 II S. 519, 520 (Änderung und Durchführungsvereinbarung).

85 Vgl. oben Teil III.1.d.

86 Vgl. z.B. Art. 12 des deutsch-österreichischen Abkommens von 1988 (Fn. 80).

87 Vgl. z.B. Art. 11 des deutsch-tschechischen Abkommens von 2000 (Fn. 80). 
men über die internationale Zivilluftfahrt ${ }^{89}$ die Vertragsstaaten, einem in Not geratenen Luftfahrzeug Hilfe zu gewähren und sich an einer koordinierten Suche nach vermißten Luftfahrzeugen zu beteiligen (Art. 25). Im Falle eines Unfalls mit der Folge des Todes oder einer schweren Verletzung von Menschen oder eines Unfalls, für den schwere technische Mängel verantwortlich sein könnten, muß der Vertragsstaat, in dessen Territorium sich der Unfall ereignet hat, eine Untersuchung in Übereinstimmung mit den Regeln der ICAO durchführen (Art. 26).

c) Ein Regelungskomplex besonders großer Bedeutung betrifft Maßnahmen bei Ölverschmutzungs-Unfällen auf Hoher See. Die entsprechenden völkerrechtlichen Regelungen gehen wesentlich auf die Erfahrung der Havarie des liberianischen Öltankers »Torrey Canyon« vor der Südwestspitze Englands im März 1967 zurück. Auf Ersuchen Großbritanniens entwarf die IMO (damals: IMCO) ein Abkommen, das im Jahre 1969 in Brüssel verabschiedet wurde und 1975 in Kraft trat $^{90}$ - das Internationale Übereinkommen über Maßnahmen auf Hoher See bei Ölverschmutzungs-Unfällen ${ }^{91}$. Dieses sog. Interventions-Übereinkommen regelt primär die Frage der Befugnisse eines Küstenstaates, zum Schutz eigener Interessen in die Rechtspositionen des Schiffseigentümers und des Flaggenstaates einzugreifen. Erstmals wurde klargestellt, daß ein Küstenstaat auch außerhalb des Küstenmeeres gegen havarierende Schiffe vorgehen darf. Mit einem Protokoll von 1973 wurden diese Interventionsbefugnisse auf Fälle von Verschmutzung durch andere Stoffe als Öl ausgedehnt. ${ }^{92}$

Das Bonner Übereinkommen zur Zusammenarbeit bei der Bekämpfung der Verschmutzung der Nordsee vom 9. Juni $1969^{93}$ war der erste völkerrechtliche Vertrag, der eine zwischenstaatliche Zusammenarbeit bei Unfällen stipulierte, die eine Verschmutzung der See durch Öl verursachen oder voraussichtlich verursachen werden; er wurde zum Vorbild für andere Regionen. Das Übereinkommen ist 1989 durch ein Folgeabkommen von 1983, das auch Verschmutzungen durch andere Schadstoffe als Öl umfaßt, abgelöst worden.$^{94}$ Das sog. Helsinki-Übereinkommen von $1992^{95}$ deckt den Ostseeraum ab. Auf Initiative des UN-Umweltprogrammes UNEP entstand eine Reihe von Regionalabkommen zum Meeresschutz. ${ }^{96}$ Sie enthalten alle sog. Notfallprotokolle, welche die Zusammenarbeit im Falle des Eintritts von Verschmutzungsereignissen regeln. ${ }^{97}$

88 Vgl. oben Teil II.

89 Vgl. oben Teil III.2.

90 Vgl. Rico Kassmann, Die Bekämpfung der Meeresverschmutzung nach Tankerunglücken Intervention und Kooperation, in: Tomuschat, Schutz der Weltmeere (Fn. 6), S. $129 \mathrm{ff}$.

91 Übereinkommen vom 29.11.1969, BGB1. 1975 II S. 139.

92 Protokoll vom 2.11.1973 über Maßnahmen auf Hoher See bei Fällen von Verschmutzung durch andere Stoffe als Öl, BGBl. 1985 II S. 596.

93 BGBl. 1969 II S. 2073.

94 Übereinkommen zur Zusammenarbeit bei der Bekämpfung der Verschmutzung der Nordsee durch Öl und andere Schadstoffe vom 13.9.1983, BGBl. 1990 II S. 71.

95 Übereinkommen über den Schutz der Meeresumwelt des Ostseegebiets vom 9.2.1992, BGBl. 1994 II S. 1397.

96 Vgl. Kassmann (Fn. 90), S. 137f., 144ff.

97 Vgl. auch auf globaler Ebene das Internationale Übereinkommen vom 30.11.1990 über Vorsorge, Bekämpfung und Zusammenarbeit auf dem Gebiet der Ölverschmutzung, BGBl. 1994 II S. 3799; dazu Kassmann (Fn. 90), S. 141ff. 
In Übereinstimmung mit der Entscheidung des IGH im Korfu Kanal-Fal ${ }^{8}{ }^{8}$ gelten heute Warn- und Informationspflichten im Falle eines Unfalls, wie sie alle genannten Abkommen (und außerdem Art. 198 der UN-Seerechtskonvention von 1982) vorsehen, als völkergewohnheitsrechtlich gesichert. ${ }^{99}$ Die Erklärung von Rio de Janeiro ${ }^{100}$ formuliert entsprechend: »States shall immediately notify other States of any natural disasters or other emergencies that are likely to produce sudden harmful effects on the environment of those States. Every effort shall be made by the international community to help States so afflicted.«

Fragen der Haftung und Entschädigung bei Öltankerunfällen sind Gegenstand des Internationalen Übereinkommens über die zivilrechtliche Haftung für Ölverschmutzungsschäden von 1969, das nunmehr in seiner durch ein Protokoll von 1992 geänderten Fassung gilt, ${ }^{101}$ sowie des ebenfalls 1992 neu gefaßten, ursprünglich 1971 verabschiedeten Internationalen Übereinkommens über die Errichtung eines Internationalen Fonds zur Entschädigung für Ölverschmutzungsschäden. ${ }^{102}$

\section{Internationale Organisationen}

So bedeutend heute die Rolle der internationalen Organisationen (unter Einschluß der internationalen Flußkommissionen) im Bereich der Verhütung von Katastrophen ist, so schwach ist sie in der unmittelbaren Bewältigung von Katastrophen. ${ }^{103}$ Sie ist in dieser Phase zumeist auf Appelle an die Staaten, Hilfe zu leisten, ${ }^{104}$ die Vermittlung von Informationen und gewisse koordinierende Maßnahmen beschränkt. Der Grund hierfür liegt schlicht in den mangelnden personellen und sachlichen Kapazitäten der Organisationen; sie verfügen nicht über in ständiger Bereitschaft stehende internationale Katastrophenschutzeinheiten. Entsprechend suchen sich die Staaten im Wege zweiseitiger Verträge die praktische Hilfe anderer Staaten im Katastrophenfall zu sichern (vgl. oben 1.a).

Die begrenzten Möglichkeiten internationaler Organisationen bei der unmittelbaren Abwehr von Katastrophen verdeutlicht beispielsweise das von der Generalkonferenz der Internationalen Atomenergie-Organisation (IAEO) im Jahre 1986 beschlossene Übereinkommen über Hilfeleistung bei nuklearen Unfällen oder radiologischen Notfällen. ${ }^{105} \mathrm{Gem}$.

98 Vgl. oben Teil II.2.

99 Vgl. auch das IAEO-Übereinkommen über die frühzeitige Benachrichtigung bei nuklearen Unfällen vom 26.9.1986, BGBl. 1989 II S. 435.

100 Vgl. oben Fn. 29.

101 BGB1. 1996 II S. 671.

102 BGBl. 1975 II S. 320; 1996 II S. 686; 2004 II S. 1290. Vgl. zu beiden Übereinkommen Knut Traisbach, Haftung und Entschädigung bei Öltankerunfällen, in: Tomuschat, Schutz der Weltmeere (Fn. 6), S. 169ff.

$103 \mathrm{Vgl}$. aber für den UN-Sicherheitsrat sogleich Teil V.

104 Vgl. z.B. die Resolution 59/217 der UN-Generalversammlung »Humanitarian assistance and rehabilitation for Ethiopia « vom 22.12.2004. Ähnliche Resolutionen verabschiedete die 59. Generalversammlung mit Bezug auf Angola, Liberia, Mosambik, Serbien und Montenegro sowie Somalia. Vgl. auch die Resolutionen 59/232 vom 22.12.2004 (El Niño Phänomen) und 59/279 vom 19.1.2005 (Tsunami-Katastrophe).

105 BGBl. 1989 II S. 441. 
Art. 2 des Übereinkommens ist die Leistung von Hilfe vornehmlich gegenseitige Aufgabe der Vertragstaaten. In Art. 2 Abs. 6 heißt es sodann in vorsichtiger und allgemeiner Form, die IAEO selbst entspreche einem Hilfeersuchen, »indem sie geeignete, für diesen Zweck bestimmte Mittel zur Verfügung stellt; das Ersuchen umgehend an andere Staaten und internationale Organisationen weiterleitet (...) und, wenn der ersuchende Staat es wünscht, die auf diese Weise verfügbare Hilfe auf internationaler Ebene koordiniert «. ${ }^{106}$

Von größerer Wirkung sind nach den dem Verfasser vorliegenden Informationen die Maßnahmen des Entwicklungsprogramms der Vereinten Nationen (UNDP), das ein »Transition Recovery Programme « begründet hat, und der Weltgesundheitsorganisation. Gem. Art. 2 Buchst. d ihrer Satzung hat sie die Aufgabe, »in Notfällen (in emergencies, dans les cas d'urgence) die erforderliche Hilfe zu leisten, wenn Regierungen darum ersuchen oder damit einverstanden sind «. Die WHO hat personelle Kapazitäten aufgebaut, um insbesondere im Falle des Ausbruchs einer Epidemie oder Endemie Wissenschaftler und Ärzte in die betroffenen Länder zu senden und dort Hilfsmaßnahmen zu unterstützen, zu initiieren und zu koordinieren. Es liegt auf der Hand, daß bei einem solchen Vergleich der Kapazitäten internationaler Organisationen die unterschiedliche Natur der zu bewältigenden Katastrophen (wie einer Seuche, eines nuklearen Unfalls oder einer Überschwemmung) in Betracht gezogen werden muß.

\section{Der »erweiterte Sicherheitsbegriff « und die Kompetenzen des UN-Sicherheitsrates}

Vor dem Hintergrund der Erfahrung des Zweiten Weltkriegs sah die UN-Charta von 1945 in der Hauptsache den »klassischen « zwischenstaatlichen Krieg als Bedrohung des Weltfriedens und der internationalen Sicherheit an. Dieser Bedrohung suchte sie mit einer organisierten Form kollektiver Sicherheit zu begegnen, deren Zentrum der Sicherheitsrat mit seinen Kompetenzen gemäß Kapitel VII der Charta bildet. Während die Charta also durchaus mit zahlreichen Bestimmungen, insbesondere Aufgabenzuweisungen an die Generalversammlung und den Wirtschafts- und Sozialrat, Katastrophen in einem umfassenden Sinn vorbeugen wollte, beschränkte sie 1945 die Möglichkeit des Einsatzes von wirtschaftlichen und militärischen Zwangsmaßnahmen auf den Zweck der Wahrung oder Wiederherstellung des Weltfriedens und der internationalen Sicherheit im engeren Sinne (Art. 39).

Im Laufe der 1990er Jahre setzte sich allerdings bei den Regierungen der UN-Mitgliedstaaten und in der Völkerrechtslehre die Ansicht durch, daß der Sicherheitsrat sowohl zur Abwehr äußerer Bedrohungen der Sicherheit eines Staates (auch der von nichtstaatlichen Akteuren wie terroristischen und kriminellen Gruppen ausgehenden) wie zur Verhütung und Beendigung schwerer Menschenrechtsverletzungen im Inneren eines Staates umfassende Kompetenzen besitzt und insbesondere politische, wirtschaftliche und militärische Maßnahmen nach Kapitel VII der UN-Charta ergreifen kann. Unter dem Stichwort »the responsibility to protect « wird zunehmend sogar eine Pflicht der internationalen Gemeinschaft angenommen, im Falle schwerer und syste-

106 Vgl. auch Art. 5 des Übereinkommens (»Aufgaben der Organisation«). 
matischer Menschenrechtsverletzungen zu intervenieren, wenn der betreffende Staat selbst zu einer Abhilfe nicht bereit oder in der Lage ist. ${ }^{107}$

Darüber noch hinausgehend, hat in jüngster Zeit in den Vereinten Nationen ein neuer, umfassender Sicherheitsbegriff (comprehensive collective security) zunehmende Anerkennung gefunden, der sowohl von neuen Bedrohungslagen wie von neuen potentiellen Urhebern von Bedrohungen geprägt ist. Eine vom UN-Generalsekretär berufene Gruppe hochrangiger Vertreter der Mitgliedstaaten (»High-level Panel on Threats, Challenges and Change«) hat in ihrem Abschlußbericht vom Dezember 2004 diesen neuen Begriff wie folgt definiert:

»Jedes Ereignis und jeder Prozeß, der zum Tod vieler Menschen oder zur Verringerung von Lebenschancen führt und der die Staaten als das tragende Element des internationalen Systems untergräbt, ist eine Bedrohung der internationalen Sicherheit. Ausgehend von dieser Begriffsbestimmung lassen sich sechs Gruppen von Bedrohungen ermitteln, mit denen sich die Welt heute und in den kommenden Jahrzehnten wird befassen müssen:

- wirtschaftliche und soziale Bedrohungen, einschließlich Armut, Infektionskrankheiten und Umweltzerstörung

- zwischenstaatliche Konflikte

- innerstaatliche Konflikte, einschließlich Bürgerkrieg, Völkermord und andere massive Greueltaten (large-scale atrocities)

- nukleare, radiologische, chemische und biologische Waffen

- Terrorismus

- grenzüberschreitende organisierte Kriminalität. « ${ }^{108}$

»Diese Bedrohungen gehen gleichermaßen von nichtstaatlichen Akteuren wie von Staaten aus und gefährden sowohl die menschliche Sicherheit als auch die Sicherheit von Staaten. « ${ }^{109}$

Der UN-Generalsekretär hat sich im März 2005 in seinem zusammenfassenden UNReformbericht »In larger freedom: towards development, security and human rights for all $\ll{ }^{110}$ dieser Auffassung angeschlossen.

Der Ausweitung des Sicherheitsbegriffs entspricht konsequenterweise eine starke Betonung der Notwendigkeit präventiver Maßnahmen, die schon im Titel des zweiten Teils des Panel-Berichts aufscheint: Collective security and the challenge of prevention. Während sich dem »klassischen« Friedensbruch effektiv mit repressiven Mitteln begegnen ließ, ist dies bei den »neuen Gefahren« nicht mehr oder nur beschränkt mög-

107 Einflußreich war in dieser Beziehung der Bericht »The Responsibility to Protect« der von der kanadischen Regierung berufenen International Commission on Intervention and State Sovereignty vom Dez. 2001.

108 A more secure world: our shared responsibility. Report of the High-level Panel on Threats, Challenges and Change, UN-Dok. A/59/565 vom 29.11.2004, Zusammenfassung des 2. Teils (Übersetzung des Deutschen Übersetzungsdienstes der Vereinten Nationen).

109 Ebd., aus der Gesamtzusammenfassung.

110 UN-Dok. A/59/2005 vom 21.3.2005. Vgl. zu beiden Berichten: Bardo Fassbender, UNReform und kollektive Sicherheit, Berlin 2005 (»Global Issue Papers« der Heinrich-BöllStiftung, Nr. 17). 
lich. Armut, Umweltzerstörung und Krankheiten lassen sich nicht durch Einsatz militärischer Gewalt lindern oder aufheben. Sie lassen sich auch nicht durch eine einzige Institution oder ein einziges Organ der internationalen Gemeinschaft verhindern, sondern nur durch einen koordinierten Einsatz verschiedener Einrichtungen und Instrumente.

Armut, Krankheiten, Umweltzerstörung und organisiertes transnationales Verbrechen als Bedrohungen des Weltfriedens und der internationalen Sicherheit anzusehen, müßte konsequenterweise dazu führen, entsprechende Kompetenzen des Sicherheitsrates nach Kapitel VII der UN-Charta anzuerkennen. Der Sicherheitsrat müßte also etwa im Falle schwerer Verletzungen oder Zerstörungen natürlicher Umweltgüter in einem Staat oder im Falle einer konsequenten Weigerung einer Regierung, Armut in geeigneter und möglicher Weise zu bekämpfen, gegen das betreffende Land Wirtschaftssanktionen verhängen oder sogar militärische Maßnahmen ergreifen dürfen. Soweit aber gehen die Autoren des Panel-Berichts nicht. Im Abschnitt über die Bekämpfung von HIV/AIDS etwa heißt es nur, der Sicherheitsrat solle in Zusammenarbeit mit UNAIDS eine zweite Sondersitzung über »HIV/AIDS as a threat to international peace and security « abhalten, um die Kenntnis des Problems zu verbessern und eine Langzeitstrategie zur Minimierung der Bedrohung zu entwickeln (Abs. 67). Indem der Bericht also für diese neuen Gefahren - nicht aber für die Gefahr des Terrorismus (vgl. Abs. 151-153), Völkermord und schwere Menschenrechtsverletzungen (vgl. Abs. 203) - auf halbem Wege stehenbleibt, hält er unausgesprochen an einer Unterscheidung von Bedrohungen des Weltfriedens nach den Maßstäben militärisch-polizeilicher Sicherheit fest.

Entsprechend wird sich der Sicherheitsrat vermutlich in der absehbaren Zukunft nicht oder in nur vorsichtiger Weise mit Katastrophen außerhalb des Bereichs KriegBürgerkrieg-Terrorismus-Völkermord befassen. Immerhin ist aber die theoretischdogmatische Arbeit schon weitgehend geleistet, die es dem Rat erlauben würde, bestimmte Formen von Armut, Umweltzerstörung und Krankheiten als Bedrohung des Weltfriedens aufzufassen und zum Gegenstand völkerrechtlich bindender Resolutionen zu machen - falls es künftig unter den Ratsmitgliedern, insbesondere den ständigen Mitgliedern, einen dahingehenden politischen Konsens geben sollte.

\section{Schlußfolgerungen}

In Anknüpfung an das in Art. 1 der UN-Charta proklamierte Ziel umfassender internationaler Zusammenarbeit der Mitgliedstaaten beschrieb Wolfgang Friedmann 1964 den mit der Gründung der Vereinten Nationen sichtbar gewordenen und verstärkten Wandel des Völkerrechts so: Das klassische »international law of coexistence « sei im wesentlichen ein »negative code of rules of abstention « gewesen - ein Kodex von an die Staaten gerichteten Verboten bestimmter Verhaltensweisen (wie der Verletzung fremden Territoriums oder fremder Staatsangehöriger oder der Einmischung in die inneren Angelegenheiten eines fremden Staates). Zu diesem Völkerrecht der Koexistenz sei - ansatzweise in der Ära des Völkerbundes, durchgreifend aber erst nach dem Zweiten Weltkrieg - ein »international law of co-operation « getreten, das den Staaten 
kooperatives Handeln gebiete, und zwar in einer Vielzahl von dem alten KoexistenzRecht noch unbekannten Sachbereichen. ${ }^{111}$ Friedmann sprach auch von einem $»$ universal interest of mankind in survival «. Dieses allgemeine Überlebensinteresse der Menschheit begründe ein Interesse an der Bewahrung der gemeinsamen natürlichen Ressourcen (in the preservation of common resources).

Die Entwicklung der völkerrechtlichen Befassung mit Katastrophen zeigt genau diesen Weg von einem Koexistenz- zu einem Kooperationsrecht. Auf der Grundlage zahlreicher zwei- und mehrseitiger Verträge, die zunächst die Hohe See und gemeinsame Flußläufe betrafen, arbeiten heute die Staaten regelmäßig und kontinuierlich zusammen, um den Eintritt von Katastrophen zu verhüten und Katastrophen im Falle ihres Eintritts praktisch besser zu bewältigen. Wie in anderen Bereichen des Völkerrechts haben sich in der zweiten Hälfte des 20. Jahrhunderts die internationalen Organisationen zu den wichtigsten Foren und Katalysatoren dieser Zusammenarbeit entwickelt. Jedoch spielen bisher die Organisationen bei der Katastrophenprävention eine weitaus bedeutendere Rolle als bei der unmittelbaren Bewältigung von Katastrophen (vgl. oben IV.2).

Noch gilt der Grundsatz der territorialen Souveränität auch für den Katastrophenschutz. Jeder Staat ist für die Verhütung und Bewältigung von Katastrophen auf seinem Gebiet primär selbst verantwortlich. Diese Verantwortung wird auch als Entsprechung zu dem »souveränen« Recht eines Staates aufgefaßt, auf dem eigenen Gebiet Handlungen vorzunehmen oder zu dulden, die eine »Katastrophenlatenz« aufweisen. ${ }^{12}$

Doch sind wesentliche Veränderungen dieser völkerrechtlichen Lage schon eingetreten oder absehbar. Die zahlreichen vertraglichen Verpflichtungen zu einer konkreten und effektiven Zusammenarbeit, welche die Staaten mit dem Ziel der Verhütung von Katastrophen auf dem eigenen Staatsgebiet übernommen haben, verdichten sich zu einer gewohnheitsrechtlichen Kooperationspflicht. Eine solche läßt sich auch aus der Verpflichtung aller Staaten zum Schutz grundlegender Menschenrechte, insbesondere des Rechts auf Leben und körperliche Unversehrtheit, der ihrer Staatsgewalt unterworfenen Personen herleiten. Mit derselben Begründung läßt sich auch vertreten, $\mathrm{da} ß$ ein Staat nach Eintritt einer Katastrophe auf seinem Gebiet ihm angebotene Hilfe anderer Staaten und internationaler Organisationen nicht ablehnen darf, wenn er nur so seinen völkerrechtlichen Schutzpflichten gegenüber der eigenen Bevölkerung gerecht werden kann.

In der Folge dauernder Befassung internationaler Organisationen, insbesondere der UN-Generalversammlung, mit Wirbelstürmen, Überschwemmungen, Erdbeben und Hungerkatastrophen hat sich ein entsprechendes Interventionsrecht der internationalen Gemeinschaft entwickelt. Kein Mitgliedstaat der Vereinten Nationen kann heute mehr behaupten, solche Katastrophen gehörten zu seinen inneren Angelegenheiten, in welche die Weltorganisation nicht eingreifen dürfe (vgl. Art. 2 Ziff. 7 der UN-Charta).

111 Vgl. Wolfgang Friedmann, The Changing Structure of International Law, London 1964, S. $60 \mathrm{ff}$.

112 Vgl. auch Prinzip 2 der Erklärung von Rio (Fn. 29). 
Das völkerrechtliche Katastrophenschutzrecht steht heute in einer engen Verbindung und Verschränkung mit dem internationalen Menschenrechtsschutz, dem Recht der Entwicklungszusammenarbeit und dem internationalen Umweltschutzrecht. Alle diese Rechtsgebiete dienen der Verhütung einschlägiger Katastrophen. Gerade das Umweltschutzrecht ist jeweils in der Folge von großen Tanker-, Chemie- und Atomunfällen (»Torrey Canyon«, »Exxon Valdez«, »Seveso«, »Bhopal«, »Tschernobyl $\ll$, die das Umweltbewußtsein schärften, weiterentwickelt worden. ${ }^{113}$

Die praktische Wirksamkeit der verschiedenen Rechtsinstrumente zur Katastrophenprävention ist nicht leicht zu beurteilen. Die aufgrund der völkerrechtlich normierten zwischenstaatlichen Zusammenarbeit vermiedenen Katastrophen werden im allgemeinen nicht bekannt. In verschiedenen Bereichen, zum Beispiel dem Schutz der Meere vor Ölverschmutzung, sind die vertraglichen Regelungen sukzessive verbessert und verfeinert worden, was für ein »learning from experience« der beteiligten Regierungen spricht. Die Einhaltung der vertraglichen Absprachen kann im allgemeinen nur im gegenseitigen Verhältnis von den Regierungen selbst überwacht werden; einzelne Verträge, insbesondere die im Rahmen von internationalen Organisationen entstandenen, weisen auch Organen dieser Organisationen Überwachungskompetenzen zu. Erzwungen werden kann die Einhaltung kaum; hier ist es bisher bei den verhältnismäßig schwachen Mechanismen des allgemeinen Völkerrechts geblieben.

Im Abschlußdokument ihres diesjährigen Gipfeltreffens aus Anlaß des sechzigjährigen Bestehens der Vereinten Nationen haben die Staats- und Regierungschefs der UNMitgliedstaaten erklärt: »We believe that today, more than ever before, we live in a global and interdependent world. No State can stand wholly alone. « ${ }^{114}$ Ohne Zweifel gilt dies auch für die Verhütung und Bewältigung großer Katastrophen.

113 Vgl. Graf Vitzthum (Fn. 12), S. 416.

114 Draft Outcome Document (Fassung vom 13.9.2005), Abs. 7. 